\title{
Е.В. КУВШИНОВА
}

\section{ИНФЛЯЦИОННАЯ МОДЕЛЬ ВСЕЛЕННОЙ С МЕТРИКОЙ ТИПА ІХ, ВОЗМОЖНОСТЬ ЕЕ КВАНТОВОГО РОЖДЕНИЯ}

\begin{abstract}
В рамках общей теории относительности построена анизотропная космологическая модель с расширением и вращением с метрикой типа IX по Бьянки. Рассматривается первая стадия инфляции Вселенной, заполненной скалярным полем и анизотропной жидкостью. В подходе, реализованном в данной модели, анизотропная жидкость описывает вращающуюся тёмную энергию. Получено уравнение Уилера - Де Витта, исследована возможность минисуперпространственного квантования.
\end{abstract}

Ключевые слова: первая стадия инфляции, темная энергия, квантовое рождение Вселенной.

\section{Введение}

В последние годы увеличилось число работ по квантовой космологии, исследуется квантовое рождение расширяющихся изотропных вселенных: закрытой, открытой и плоской [1-4]. Вместе с тем в работе [5] найдена волновая функция для однородной изотропной модели Вселенной с электромагнитным полем, чистым излучением и вращающейся жидкостью, классическое поведение которой описывается космологическим решением Вайдьи и Пателя. Авторы работы [5] делают вывод, что космологические состояния с большими величинами глобального вращения являются маловероятными. В [6] в рамках формализма суперпространственного квантования рассматривается квантовая эволюция однородных вращающихся космологических моделей типа Гёделя со спинорным и скалярным полями. Там показано, что уравнение Де Витта для вращающихся космологических моделей принимает форму уравнения Шредингера, в котором роль времени играет фаза спинорного поля, и возникает возможность корректного определения вероятности существования вращающихся моделей, у которых отсутствует начальная сингулярность. Квантовое рождение Вселенной с медленным вращением исследовано в $[7,8]$, причем авторы этих работ в рамках своего подхода установили, что вращение может как уменьшать, так и увеличивать коэффициент туннелирования Вселенной. Квантовое рождение Вселенной с произвольным вращением рассматривалось в [9-11]. Исследование роли вращения в описании ранней Вселенной и при квантовом рождении Вселенной представляется актуальным. В данной работе исследуется возможность квантового рождения модели Вселенной с метрикой типа IX по Бьянки.

\section{Модель Бьянки IX}

В данной работе в рамках общей теории относительности найдено решение с вращением на основе метрики типа IX по Бьянки вида

$$
d s^{2}=\left(d t+A \omega^{1}\right)^{2}-\left(B \omega^{1}\right)^{2}-C^{2}\left(\left(\omega^{2}\right)^{2}+\left(\omega^{3}\right)^{2}\right),
$$

где $A, B, C$ - функции, зависящие от времени; $\omega^{1}, \omega^{2}, \omega^{3}-1$-формы, удовлетворяющие структурным отношениям типа IX по Бьянки.

Представим нашу метрику в тетрадной форме. Используется лоренцевая тетрада с ненулевыми компонентами:

$$
\begin{gathered}
e_{0}^{(0)}=1, \quad e_{1}^{(0)}=-A \sin x^{3}, \quad e_{2}^{(0)}=A \sin x^{1} \cos x^{3}, \\
e_{1}^{(1)}=-B \sin x^{3}, \quad e_{2}^{(1)}=B \sin x^{1} \cos x^{3}, \\
e_{1}^{(2)}=C \cos x^{3}, \quad e_{2}^{(2)}=C \sin x^{1} \sin x^{3}, \\
e_{2}^{(3)}=C \cos x^{1}, \quad e_{3}^{(3)}=C .
\end{gathered}
$$

Применяя метод, предложенный в [12], найдем условия, которые обеспечивают причинность пространства-времени с метрикой (1). Пусть $x^{\mu}(s)$ - произвольная времениподобная кривая $(s-$ параметр), $v^{\mu} v_{\mu}>0$. Если предположить, что эта кривая - замкнутая, тогда всегда существует та- 
Уважаемые читатели!

Доступ к полнотекстовой версии журнала «Известия высших учебных заведений. Физика» осуществляется на платформе Научной электронной библиотеки ELIBRARY.RU на платной основе:

https://www.elibrary.ru/contents.asp?titleid=7725 